INTERNATIONAL DESIGN CONFERENCE - DESIGN 2018

https://doi.org/10.21278/idc.2018.0540



\title{
VERBAL ENGAGEMENT IN TEAMS SOLVING A CONCEPTUAL DESIGN TASK
}

\author{
T. Martinec, N. Horvat, S. Škec and M. Štorga
}

\begin{abstract}
The paper represents an explorative step in studying the effect of problem-solving styles on verbal engagement in teams solving a conceptual design task. The selection of VIEW problem-solving style assessment framework provided assumptions on how different style dimensions could affect verbal engagement. The assumptions were tested by setting up a protocol study where teams composed of members with different problem-solving styles solved a conceptual design task. The results of the protocol analysis have revealed the potential effects and provided directions for further research steps.
\end{abstract}

Keywords: design teams, conceptual design, protocol analysis, verbal engagement, problem solving

\section{Introduction}

Conceptual design is often regarded as a critical development phase, not just in engineering design, but also in other design domains. Concepts, just as products, have to be designed and described in a way others can understand them and build on them (Andreasen et al., 2015). The activities of developing concepts are potentially the most creative, vibrant and dynamic from the designers' point of view, but also the least understood from the researchers' perspective (Macmillan et al., 2001). There is, however, general agreement that organisations benefit from utilising teams to harness the creative potential in concept development. Due to the nature of design problems, conceptualisation requires understanding and decomposition of the given design problem, before viable concept solutions could be proposed (Liikkanen and Perttula, 2009). Ideally, team members should encourage and inspire each other to generate, analyse and evaluate more design content in both problem and solution space, to produce better and comprehensive conceptual solutions. Accordingly, conceptual design tasks such as idea-generation and concept selection are often performed explicitly in teams (Sonalkar et al., 2013).

There exists a number of measures for assessing the contribution of team members during design activities. Assessments include measuring the characteristics of the inputs - what individuals bring to the team (knowledge, skills, abilities, motivation, attitude, personality), and the outputs - the design content individuals have produced (product performance, creativity, novelty and satisfaction), but also measuring different aspects of the process - how individuals create (ideation, sketching, prototyping, mental models) (Takai and Esterman, 2017).

Although there is a number of factors influencing teamwork in design, it can be argued that both the process and outputs of team conceptual design tasks will to a large degree depend on the inputs individual characteristics of team members taking part in the task (Birdi et al., 2016). Studies on the individual characteristics of designers are usually based on personality assessments (e.g. Myers-Briggs Type Indicator - MBTI) and cognitive style assessments (e.g. Kirton Adaption-Innovation Inventory KAI) which have shown to affect the process of designing in teams (see e.g. Peeters et al., 2008; and 
Sonalkar et al., 2017). Besides that, an individual's preferred way of action when dealing with problems (i.e. problem-solving style) has shown to influence both the design process and the design outcome (Eisentraut, 1999). Thus, the design process is often referred as a problem-solving process (BadkeSchaub and Buerschaper, 2001). Hence, identification of the dependencies between problem-solving style and team conceptual design process is necessary for better understanding of both designers' contribution in team tasks and team conceptual design process as such.

Here presented work is an exploratory step in the research aimed to investigate the effects of individuals' problem-solving style on the process of a team conceptual design task. Design content produced by teams solving conceptual design tasks consists mostly of formulated problems and generated solutions. The communication of such content between team members is primarily verbal (Andreasen et al., 2015), usually backed up by visual representations for improved shared understanding (Nik Ahmad Ariff et al., 2012). Capturing verbal acts during conceptual design sessions can thus provide a basis for analysing team members' processes and contribution. For this reason, researchers often employ verbal protocol analysis - a method that relies on verbal expressions of the participants - to analyse and understand the underlying cognitive processes of designers. Consequently, the verbal engagement (often regarded to as verbal participation or, simply, time spent talking) of designers can, to some extent, be a proxy of their overall contribution (DiMicco et al., 2004).

The focus of here presented research is set on exploring the verbal engagement of individuals characterised by different problem-solving styles when they are conceptualising in teams. Two main research questions guided the study:

- Does the problem-solving style of individuals affect their verbal engagement when solving conceptual design tasks in teams?

- Does the combination of team members' problem-solving styles affect the team-level verbal engagement?

Research questions have been addressed by introducing assumptions on the verbal engagement of team members based on the theoretical background on problem-solving in design. Assumptions are followed by the description of experimental setup and verbal protocol analysis as the method for investigating the assumptions. Lastly, the results of the protocol analysis are presented and discussed, and new insights and future directions are pointed out.

\section{Background}

\subsection{Design as problem-solving}

Before exploring the effects of different problem-solving styles, it is necessary to understand the perception of problem-solving in design. There is a number of perspectives on design as problemsolving, some of which are presented in the following paragraphs.

Conventional problem solving is seen as a recurring and dominant model of design used in design education, but also as one which has been working well in many professions, especially in engineering design where the goals are clear, explicit and stable (Lawson and Dorst, 2009). This may be the reason why the engineering design textbooks have well embraced the conventional problem-solving perspective of designing. For example, Pahl and Beitz (1996) propose a general problem-solving process and suggest that each design stage is a journey through a same problem-solving cycle. Similarly, Ullman (2010) identifies conventional problem-solving steps regardless of the type of design problem. The steps include planning of the process, understanding the problem, generating and evaluating solutions, deciding on acceptable solutions and communicating the results. Dieter and Schmidt (2013) provide a very much alike problem-solving methodology which can be used at any point in the design process. Andreasen and Hein (1987) explain that a basic sequence of a conventional problem-solving approach appears at many levels in the design process, including the stages as the top-level.

Unlike the prescriptive design methodologies, the research streams which attempt to model how problem-solving in design is conducted in reality mostly struggle to identify the structure of conventional problem-solving, due to the multifaceted nature of design (Lawson and Dorst, 2009). Stempfle and Badke-Schaub (2002) consider designing as a specific area of problem-solving, as both 
require that the solutions meet all of the relevant requirements. They have been able to identify some of the conventional problem-solving steps within the thinking processes of designers. Kurakawa (2004) based his conceptual design model on the cognitive design problem-solving process, which shares the essence of the conventional problem-solving in product design. He assumes that this process in which designers engage during design meetings is the most basic process governing design.

Schön's observations of the design practice made it clear that conventional technical problem solving is an incomplete description of what engineers do (Schön, 1983). He explains that this is especially noticeable when practitioners address new or unique problems that do not fit known solving procedures. Not only can design problems be new or unique, but they are also often ill-defined. Cross (2001) explains that when faced with ill-defined problems, designers typically do not start by pursuing to define the problems rigorously, but rather progressively define the problem in relation to solution ideas they are developing. This is in contrast to the conventional problem-solving approaches. Hansen et al. (2009) have encountered the incompleteness issues of conventional problem-solving models through their experience in design teaching and design practice. They describe that the incompleteness is particularly evident during the problem formulation step where teams' processes are discursive rather than a straightforward problem-solving.

It can be argued that for the large part, the researchers' descriptions of designing depend on the given design problem, where some problems are tackled through a conventional problem-solving, while others require problems and solutions to evolve in parallel. The viewpoint on the design by Dorst can complement these two distinctive perspectives of problem-solving. He introduces the notion of abduction - the basic reasoning pattern in productive thinking which comes in two forms (Dorst, 2011). The first which is often associated with conventional problem-solving, where both the value to be created and the working principle is known, and what is missing is the object/service/system that will define both the problem and the solution space. The second form, which is more closely associated with (conceptual) design is more complex, as only the end value is known and the challenge is to figure both what to create (object/service/system) and how it will achieve the value (working principle) (Dorst, 2011).

\subsection{Problem solving style}

Due to the problem-solving perspective that has been employed throughout the paper, the individual preferences on conceptual designing have been addressed as problem-solving styles. Problem-solving styles do not reflect the ability, skills or creativity, but they describe the way designers prefer to solve and deal with problems. Unlike behaviour which is flexible, problem-solving styles are stable, and resistant to change (Eisentraut, 1999; Kirton, 2003). The observed behaviour of individuals is sometimes in accord with preferred style, while other times when designers work outside their preferred style, the coping behaviour activates, requiring extra effort and introduces stress experience (Jablokow, 2005).

To focus solely the problem-solving styles, the VIEW model of problem-solving style has been selected as the framework for the purpose of this study. The VIEW model was not used by previously conducted research in the design science, but since it has been specifically developed from the theory of creative problem-solving (Selby et al., 2014) - the same process which can describe designing (Badke-Schaub and Buerschaper, 2001), it presented a reasonable framework for this study. Moreover, the model has been related to other psychometric measures such as KAI inventory (Treffinger et al., 2014) - a commonly used measure in the design science which allows direct comparison with the other studies in the field. VIEW framework highlights the difference in styles between team members in three dimensions: Orientation to Change, Manner of Processing and Ways of Deciding. The scores reflect individuals' preferences which are emphasised through their typical behaviour across varying context and over sustained periods of time - the farther their score from the mean, the more clear, certain and consistent are their preferences (Treffinger et al., 2008).

\subsubsection{Orientation to Change}

The first dimension, Orientation to Change (OC), provides an overall indication of individual's perceived preferences and dispositions for dealing with novelty and originality, for structure and authority and for searching for data, options and understanding. The style in this dimension falls along a continuum ranging from Explorer to Developer (Selby et al., 2014). 
- Explorers initiate a wide range of tasks and thrive on new, ill-defined and ambiguous situations and challenges. They often find imposed plans, structures and procedures as limiting and confining (Treffinger et al., 2008).

- Developers prefer problems and solutions to be within the framework of present experience and seek incremental, practical and easily assimilated changes. Developers' concern is with the practical applications and the reality of the task (Treffinger et al., 2008).

These two styles are of significant interest as they seem to distinguish the two types of abduction suggested by Dorst (2011) - characteristics of conventional problem-solving can be identified in preferences of developers, and characteristics of addressing the problems in new frames can be identified in preferences of explorers. A similar distinction exists within the KAI framework, where adaptors prefer more structure and working within given problems and innovators prefer less structure and strive to transcend given frames (Kirton, 2003). There is also evidence of a significant correlation between the OC dimension score and KAI score (both on overall subscale level) (Treffinger et al., 2014).

\subsubsection{Manner of Processing}

Manner of Processing (MP) dimension relates to the extraversion and introversion constructs in other frameworks, but rather than characterising it as a type of personality, it focuses on individual's behavioural preferences when they solve problems or manage change. MP addresses the use of personal inner energy and resources, the energy and resources of others and the environment. Styles fall along a continuum ranging from External to Internal (Selby et al., 2014). As expected, there exists a significant correlation between external/internal styles and extraversion/introversion personalities in MBTI (Treffinger et al., 2014).

- Externals draw energy by interacting, discussing and building from the ideas of others. When solving problems, they test their views against those of others and seek input from others. They prefer active and lively idea exchange and may also dominate idea generation sessions (Treffinger et al., 2008).

- Internals draw energy from their inner reflection and get preoccupied with inner events, ideas and concepts. In contrast to externals, they may seem quiet and be perceived as withdrawn. They prefer quiet reflection and processing information at their own pace (Treffinger et al., 2008).

The preference on information processing is of interest because of its reflection on verbalisation, as externals styles tend to engage more verbally than the internals. Furthermore, MP dimension could potentially be correlated with creativity, as studies have shown a positive correlation between MBTI's extraversion and creativity in the engineering domain (Yan et al., 2013).

\subsubsection{Ways of Deciding}

The third dimension, Ways of Deciding (WD), deals with individual's balancing of task concerns with personal or interpersonal needs. This dimension focuses on the factors that get priority when deciding, on the preferred starting point and how trade-offs are made. The style in this dimension falls along a continuum ranging from Person to Task (Selby et al., 2014).

- Individuals with person preference care for harmony and positive relationships and therefore consider first the impact of decisions on other people's feelings and support. During idea generation, they try to ensure that all ideas are respected, that all concerned are heard, and seek decisions that satisfy most of the team (Treffinger et al., 2008).

- Individuals with task preference tend to make decisions that are logical, sensible and can be justified objectively, and therefore make judgements that are impersonal, free of emotion and based on well-reasoned conclusions. During idea generation, they often develop clear plans of actions and offer strong leadership in identifying the most promising and realistic options (Treffinger et al., 2008).

Person- and task-focused individuals could thus differ in the content of contribution, especially when applying evaluation operations and when a decision has to be made. 


\subsection{Verbal engagement}

The initial step in the exploration of the dependency between team members' problem-solving styles and the process of solving a conceptual design task is identifying the effects of problem-solving style on the verbal engagement of each team member. There are two reasons for this being the first step. First is that verbal segmentation is the first dataset available when conducting protocol analysis, and second is the importance of verbal engagement as reported in different domains.

Although verbal engagement has mostly been studied within education research as a measure of students' engagement in class activities, there have been efforts to recognise its role in team discussions. The main focus of such studies was on leadership. For example, Willard and Strodtbeck (1972) give an overview of studies showing a strong relation between individual's amount of engagement in small group discussion and their leadership or influence in the group. Studies have found high correlations between participant's verbal engagement and their rank on productivity as perceived and ranked by other group members. Philipsen and Saine (1973) have demonstrated the effect of reward criteria on both frequency and ratio of team-centred interactions when solving problems. Sorrentino and Field (1986) have shown high correlations between the quantity of verbal engagement and leadership in group problem-solving activities. They also confirmed the previous reasoning that verbal engagement appears to be a strong indicator of individual's strong motivation to help the group to solve its goal.

Research approaches in both, engineering practice and education have been focused on different factors influencing participation (not only verbal) in small groups, such as inter-group distribution of gender, ethnicity, students' grades and the influence of different engineering programs (Ohland et al., 2008; Dasgupta et al., 2015). As for the measurement and analysis of verbal engagement, it is worth to mention Temporal Static Visualizations, a tool for analysing team meeting transcripts and exposing actual communication flow and the historical participation of team members (Wulvik et al., 2017).

\subsection{Study assumptions}

Relating problem-solving style to verbal engagement in conceptual design sessions could increase the understanding and the ability to predict the behaviour of different team compositions engaged in team conceptual design activities. Following the research questions, several assumptions are proposed based on the insights presented above:

- First assumption is that team members with lower OC (explorers) tend to have higher verbal engagement rate than team members with higher OC score (developers) when solving conceptual design tasks. This assumption is based on the characterization of conceptual design problems as ill-defined. Explorers' preference to solve new, ill-defined and ambiguous situations and challenges should help them to engage more easily than developers.

- Second assumption is that team members with lower MP (externals) tend to have higher verbal engagement rate than team members with higher MP score (internals) when solving conceptual design tasks. This assumption is intuitive, as externals are described to prefer interaction, discussion and active and lively idea exchange, unlike internals who prefer quiet reflection.

- Third assumption is that team members with higher WD (task-focused) tend to have higher verbal engagement rate than team members with lower WD (person-focused) during conceptual design task sessions. This assumption is based on the association of leadership with verbal engagement. Individuals with task preference provide strong leadership, show task commitment and develop clear plans of actions during idea generation.

\section{Methodology}

The empirical part of the research has been designed in the form of a verbal protocol analysis aimed to address the proposed assumptions. Because team members naturally communicate verbally as they work together, recordings of team discussions are authentic verbal outputs of real-time thinking (Goldschmidt, 2014). Therefore, verbal protocol analysis is a practical and a valid method for analysing teamwork sessions. 
The study was conducted in several steps. First, the participants of the study were selected, and their problem-solving styles were assessed. The study participants were grouped into teams and given a conceptual design task to solve during a team session. The recordings from these sessions were segmented and analysed to provide data results for discussion.

\subsection{Participant selection and problem-solving style assessment}

Selecting participants is based on the explorative aim of this study to observe the relationship of problem-solving style and verbal engagement. For this type of study, a laboratory experiment is appropriate since it can be highly constrained (Dinar et al., 2015) and they do not show different conversational activities when compared to real practice observation (Cash et al., 2013). Laboratory experiments are also convenient since they usually use students as participants (Cash et al., 2013). Hence, participants were selected among third- and fourth-year students of mechanical engineering, who have previously been enrolled in courses such as Product development, Design methods and Advanced computer-aided design (CAD). The students also finished a one-semester project-based learning course, where they were engaged in the realisation of a complex mechatronic device. The product realisation project included the phases of planning, conceptual and detail design, and prototype assembly and testing. Such background provided students with experience in both working in teams and developing conceptual solutions.

A problem-solving style assessment workshop was organised for the experiment participants. The workshop was led by a qualified professional, trained in the application, feedback, and administration of VIEW. In the first part of the workshop, the 16 participants were introduced to the concept of psychometric testing and the role which psychometric assessments can play in the selection and deployment of creative groups/teams. Their problem-solving style was assessed using a current edition VIEW assessment instrument, which consists of 34-items - 18 items dedicated to OC, eight to MP, and eight to WD. All items were scored from 1 to 7. The scores in the OC dimension for the participants range from 18 to 126, and in the MP and WD dimensions from 8 to 56. A complete overview of the participants' scores across the three dimensions is available in Table 1.

The 16 available participants were mixed into six three-member teams, with the aim of having differences in VIEW dimensions within the team and in-between teams, since it is expected that the relative difference in team members' scores will distinguish their observable preferences.

Inter-team differences were ensured by calculating average VIEW scores of team members. The distribution of average scores is available in Table 2.

Differences within the teams were ensured by calculating relative VIEW scores which place the problem-solving styles of team members in the context of their teams. The relative VIEW score in each dimension has been calculated as differences between individual score and the average score of their team. Thus, the more positive or negative relative OC score of a participant, the more clearer developer or explorer characteristics they have compared to the team. Positive and negative relative MP scores distinct internal and external processing team members (in that order), while positive and negative relative WD scores distinct person-focused and task-focused team members. Relative VIEW scores are shown in Table 1.

Four experiment sessions had a constant formation (team formations 1-4), and one had two formations (team formations $5 \mathrm{a}$ and $5 \mathrm{~b}$ ) - after the first half of the session, one team member was replaced with a new one of the different style to inspect if such change affects verbal engagement.

\subsection{Conceptual design task}

The experiment was designed in the form of a 60-minute conceptual design session during which teams had to propose a concept solution for the given design problem. The design brief was ill-defined and listed only the main design problems that had to be solved. The task was to come up with a concept of a portable key-organising solution to help users deal with everyday key usage. Examples of the given requirements are illustrated in Figure 1.

Since keys are artefacts used on a daily basis, the participants should be able to understand the problem without the need for any information gathering tools (computer with internet connection, books, etc.). The proposed solution should be in the form of detail sketches of the concept, including the approximate 
dimensions, materials and handling scenarios. Participants were encouraged to work on the development of multiple solutions but to indicate the final concept proposal. However, they were not briefed on which teamwork aspect will be observed to reduce the possible influence on the teamwork.

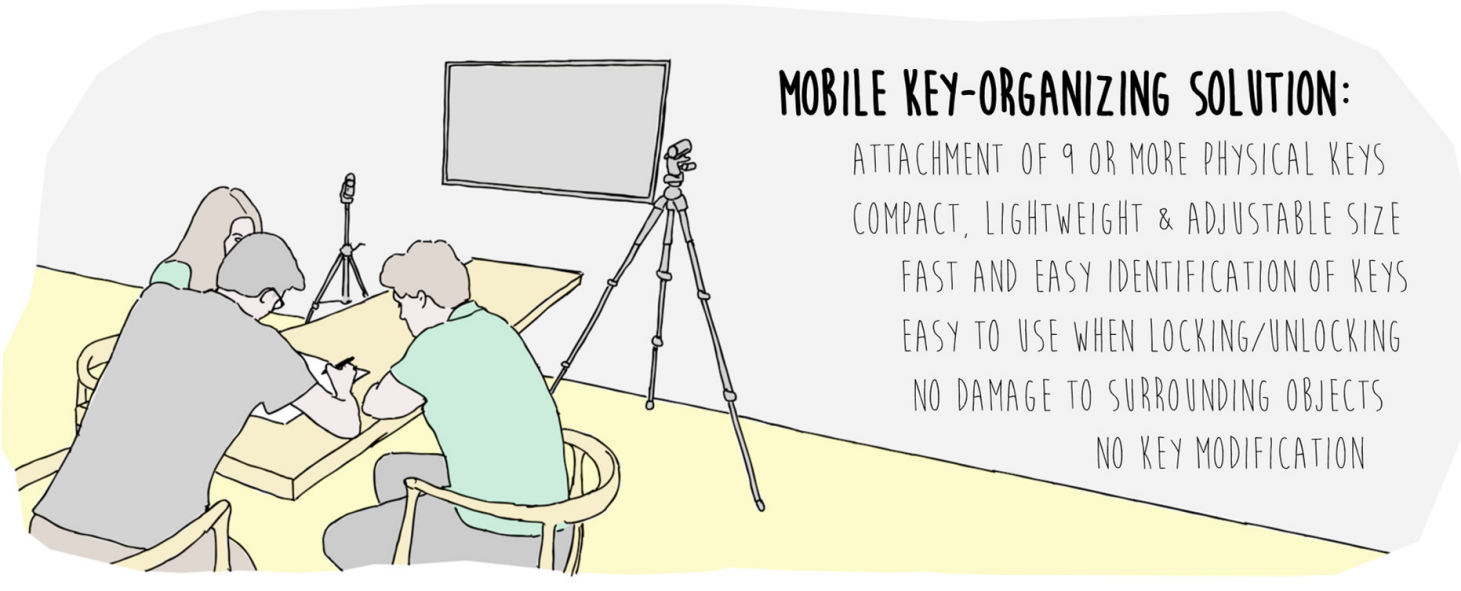

Figure 1. Experimental set-up with examples of design brief requirements

Each team was left alone in a room equipped with the recording equipment: two cameras recording video, one voice recorder for audio, and one digital notebook for recording of sketching. Participants could use a ball point pen to write in the digital notebook. Design brief was given on a wall-mounted screen and could thus be consulted at any time. The screen also included a timer so they would not have to keep track of time. Teams did not receive any information on the design task until they were left in the room and until the brief appeared on the screen. An illustration of the experimental set-up is available in Figure 1. After the session was finished, the team presented and explained in details their final concept proposal in front of the camera.

To reveal possible weaknesses of the presented approach, the experiment was firstly tested with a pilot study in which a test team conducted a design session on the described task. However, the pilot study did not reveal any major problems, so the same approach was used for teams whose communication was then further segmented.

\subsection{Segmentation}

Once all teams finished their experiment sessions, the captured recordings were synchronised, segmented and, finally, transcribed. The analyses presented in this paper is focused on the data originating from segmentation of team members' verbal engagement. All segments with verbal acts were assigned to codes based on the participant talking. The start and the end of the coded segment have been determined with the start and the duration of the participant's statement. A situation when two or three participants were talking at the same time, although rare, would be assigned with multiple codes. Verbal acts that were not coded represent periods without verbal engagement (silence).

\section{Results and discussion}

The duration of all verbal acts for each participant have been aggregated and divided by the total duration of the session to calculate the verbal rate of every participant in the form of seconds spoken per minute. To observe participants' verbal engagement in the context of their teams, the relative verbal rates were calculated by dividing individual verbal rates with the total verbal rate of the team (sum of all three team members verbal rates). Hence, relative verbal rates represent the distribution of verbal engagement of team members in the context of their team's session. The higher the percentage of relative verbal rate, the more engaged the team member was. 
Table 1. Overview of individual VIEW scores of all team members and their verbal rates across all team formations

\begin{tabular}{|c|c|c|c|c|c|c|c|c|c|}
\hline \multirow{2}{*}{$\begin{array}{l}\text { Team } \\
\text { form. }\end{array}$} & \multirow[t]{2}{*}{ Participant } & \multicolumn{3}{|c|}{ VIEW scores } & \multicolumn{3}{|c|}{ Relative VIEW scores } & \multirow{2}{*}{$\begin{array}{c}\text { Verbal rate } \\
\text { [s/min] }\end{array}$} & \multirow{2}{*}{$\begin{array}{c}\text { Relative verb } \\
\text { rate [\%] }\end{array}$} \\
\hline & & OC & MP & WD & $\mathbf{O C}_{\text {rel }}$ & MPrel & WD $_{\text {rel }}$ & & \\
\hline \multirow[t]{3}{*}{ Team 1} & $\mathrm{P} 1$ & 81 & 26 & 32 & 6.00 & -4.00 & 2.67 & 12.50 & 26.1 \\
\hline & $\mathrm{P} 2$ & 76 & 34 & 19 & 1.00 & 4.00 & -10.33 & 15.15 & 31.6 \\
\hline & P3 & 68 & 30 & 37 & -7.00 & 0.00 & 7.67 & 20.25 & 42.3 \\
\hline \multirow[t]{3}{*}{ Team 2} & $\mathrm{P} 4$ & 88 & 31 & 52 & 8.00 & 4.00 & 0.33 & 20.73 & 35.3 \\
\hline & P5 & 77 & 26 & 51 & -3.00 & -1.00 & -0.67 & 17.77 & 30.3 \\
\hline & P6 & 75 & 24 & 52 & -5.00 & -3.00 & 0.33 & 20.17 & 34.4 \\
\hline \multirow[t]{3}{*}{ Team 3} & $\mathrm{P} 7$ & 91 & 34 & 42 & 2.33 & 4.33 & 1.33 & 13.46 & 22.6 \\
\hline & P8 & 93 & 33 & 33 & 4.33 & 3.33 & -7.67 & 21.83 & 36.6 \\
\hline & P9 & 82 & 22 & 47 & -6.67 & -7.67 & 6.33 & 24.38 & 40.9 \\
\hline \multirow[t]{3}{*}{ Team 4} & P10 & 40 & 54 & 51 & -15.00 & 15.33 & 11.67 & 21.80 & 38.5 \\
\hline & P11 & 56 & 24 & 35 & 1.00 & -14.67 & -4.33 & 13.58 & 24.0 \\
\hline & $\mathrm{P} 12$ & 69 & 38 & 32 & 14.00 & -0.67 & -7.33 & 21.22 & 37.5 \\
\hline \multirow[t]{3}{*}{ Team 5a } & $\mathrm{P} 13$ & 60 & 31 & 34 & -10.00 & -1.67 & -4.00 & 27.07 & 49.6 \\
\hline & P14 & 72 & 34 & 41 & 2.00 & 1.33 & 3.00 & 15,39 & 28.2 \\
\hline & P15 & 78 & 33 & 39 & 8.00 & 0.33 & 1.00 & 12.15 & 22.3 \\
\hline \multirow[t]{3}{*}{ Team 5b } & P13 & 60 & 31 & 34 & -5.00 & -1.33 & -3.67 & 22.62 & 40.1 \\
\hline & P14 & 72 & 34 & 41 & 7.00 & 1.67 & 3.33 & 11.77 & 20.8 \\
\hline & P16 & 63 & 32 & 38 & -2.00 & -0.33 & 0.33 & 22.07 & 39.1 \\
\hline
\end{tabular}

OC - Orientation to Change; MP - Manner of Processing; WD - Ways of Deciding

Table 2. Overview of average VIEW scores and verbal rates of all team formations

\begin{tabular}{ccccc}
\hline Team & \multicolumn{3}{c}{ Average VIEW scores } & Average verbal rate \\
\cline { 2 - 4 } formation & $\mathbf{O C}_{\text {avg }}$ & MP $_{\text {avg }}$ & WD $_{\text {avg }}$ & {$[\%]$} \\
\hline Team 1 & 75.00 & 30.00 & 29.33 & 26.57 \\
Team 2 & 80.00 & 27.00 & 51.67 & 32.60 \\
Team 3 & 88.67 & 29.67 & 40.67 & 33.13 \\
Team 4 & 55.00 & 38.67 & 39.33 & 31.43 \\
Team 5a & 70.00 & 32.67 & 38.00 & 30.37 \\
Team 5b & 65.00 & 32.33 & 37.67 & 31.37 \\
\hline
\end{tabular}

The analysis of dependency between problem-solving styles and verbal engagement has been conducted on individual and team level, with a remark that the results are of explorative nature, due to a limited number of teams studied. Dependency was analysed using Pearson correlation and regression analysis. Correlation between VIEW scores and individual and team level verbal rates are shown in Table 3.

Table 3. Correlation between VIEW scores and verbal rates

\begin{tabular}{|c|c|c|c|}
\hline \multicolumn{3}{|c|}{ Individual level } & \multirow{2}{*}{$\begin{array}{l}\text { Team level } \\
\text { Average verbal rate }\end{array}$} \\
\hline & Verbal rate & Relative verbal rate & \\
\hline $\mathbf{O C}_{\text {rel }}$ & $\mathrm{r}=-0.531, \mathrm{p}=0.023^{*} *$ & $\mathrm{r}=-0.566, \mathrm{p}=0.014 * *$ & $\mathrm{r}=0.196, \mathrm{p}=0.710$ \\
\hline MPrel & $\mathrm{r}=0.088, \mathrm{p}=0.728$ & $\mathrm{r}=0.097, \mathrm{p}=0.701$ & $\mathrm{r}=-0.038, \mathrm{p}=0.943$ \\
\hline WD $_{\text {rel }}$ & $\mathrm{r}=-0.017, \mathrm{p}=0.946$ & $\mathrm{r}=-0.009, \mathrm{p}=0.971$ & $\mathrm{r}=0.798, \mathrm{p}=0.057^{*}$ \\
\hline
\end{tabular}


To analyse also the possible effects of interactions between relative VIEW scores (independent variables), multiple linear regression models have been created for verbal rates and relative verbal rates (dependent variables). Due to the limited number of team formations observed, regression analysis was performed on the individual level only. Details of these models are provided in Table 4.

Table 4. Multiple linear regression coefficients for verbal rate modelling

\begin{tabular}{|c|c|c|c|c|c|c|}
\hline \multirow[t]{2}{*}{ Variable } & \multicolumn{3}{|c|}{ Verbal rate } & \multicolumn{3}{|c|}{ Relative verbal rate } \\
\hline & B & Error & $\boldsymbol{\beta}$ & B & Error & $\boldsymbol{\beta}$ \\
\hline $\mathbf{O C}_{\text {rel }}$ & -0.499 & 0.135 & $-0.787 * * *$ & -0.895 & 0.238 & $-0.814 * * *$ \\
\hline $\mathbf{M P}_{\text {rel }}$ & 0.193 & 0.189 & 0.248 & 0.251 & 0.332 & 0.185 \\
\hline $\mathbf{W D}_{\text {rel }}$ & -0.266 & 0.180 & -0.319 & -0.447 & 0.318 & -0.309 \\
\hline $\mathbf{O C}_{\text {rel }} \times \mathbf{M P}_{\text {rel }}$ & 0.096 & 0.049 & 0.151 & 0.106 & 0.086 & 0.096 \\
\hline $\mathbf{O} \mathbf{C}_{\text {rel }} \times \mathbf{W} \mathbf{D}_{\text {rel }}$ & -0.066 & 0.025 & $-0.085^{* *}$ & -0.101 & 0.045 & $-0.074 * *$ \\
\hline \multirow[t]{2}{*}{$\mathbf{M} \mathbf{P}_{\text {rel }} \times \mathbf{W} \mathbf{D}_{\text {rel }}$} & 0.038 & 0.047 & 0.046 & 0.007 & 0.083 & 0.005 \\
\hline & \multicolumn{3}{|c|}{ Model: $\mathrm{R}^{2}=0.634 ; \mathrm{F}(6,11)=3.177^{* *}$} & \multicolumn{3}{|c|}{ Model: $\mathrm{R}^{2}=0.622 ; \mathrm{F}(6,11)=3.015^{*}$} \\
\hline
\end{tabular}

\subsection{The effect of Orientation to Change}

The individual-level calculation of Pearson's product moment correlation coefficient identified a significant correlation $(\mathrm{p}<0.05)$ between the relative OC score and both the verbal rate $(-0.529)$ and relative verbal rate (-0.566). The effect of relative OC score was confirmed as significant using multiple linear regression modelling. These significant negative correlations are aligned with the assumption that team members with lower OC (team explorers) tend to have higher verbal engagement rate than team members with higher OC score (team developers) when solving the conceptual design task.

Because of the significant and strong, negative correlation between the OC dimension score and the overall KAI score (Treffinger et al., 2014), it is possible to compare these findings with studies that used KAI assessment. Although none of these studies measured verbal engagement, they did deal with the contribution of team members. For example, in their study of engineering design teams, Sonalkar et al. (2017) argue that individuals with higher KAI score are more likely to propose a larger number of unique ideas in the team ideation sessions. Jablokow et al. (2015) have found that individuals with higher KAI score tend to feel they contributed more and that their ideas were more elaborated, while individuals with lower KAI score tend to feel the opposite. Higher verbal engagement of team members gravitating to the explorer style (correlated with higher KAI score) favours the findings reported in these studies.

The significant correlation between relative OC score and verbal engagement can also be explained due to the nature of conceptual design tasks. A study by Isaksen and Geuens (2006) reported that explorers have significantly higher levels of enjoyment for both, problem framing and generating ideas - which were identified as two important elements of conceptual design.

As for the team-level analysis, no significant correlation between average OC of the team and average verbal rate of the team was found. The reason for this could be the relatively small number of team formations studied.

\subsection{The effect of Manner of Processing}

Neither the correlation analysis or the regression analysis revealed significant effects of the relative MP score and the verbal rates of team members. This is surprising since it is the MP dimension that describes individuals' preference for interaction and discussion. Several potential reasons of why there was no significant difference in the verbal engagement of internals and externals have been identified. Firstly, the small team size reduces the effect of extraversion and introversion, as smaller teams have shown to have better developed internal relations (Kuipers et al., 2009), which include activities that bind team member and giving feedback to one another. The other reason could lie in the experience of working together that the participants gained during the one-semester project-based course (resulting in more comfort when working together in teams). Finally, no significant difference might be due to the type and the duration of 
the given task which required only a temporal effort of interaction. The effect of MP on verbal engagement might emerge over longer periods of time when constant effort of team discussion is demanded.

Considering the significant correlation between MP styles and extraversion personality in MBTI it is possible to align these results with other studies of design team meetings which, likewise, failed to provide evidence of extraversion affecting designing or design outcomes (e.g. Carr et al., 2002; Varvel et al., 2004).

\subsection{The effect of Ways of Deciding}

Correlation analysis and regression analysis did not reveal a significant effect of relative WD score on verbal rates of team members. Regression analysis, however, revealed a significant effect $(\mathrm{p}<0.05)$ of interaction between relative OC and relative WD scores. This interaction can be interpreted as an indirect effect of team members' WD preference on their verbal engagement. The effective coefficient of the interaction is negative, indicating that the explorer's verbal engagement is amplified by positive relative WD scores, which is a reflection of task-focused team members. The explorer's verbal engagement is accordingly decreased when the relative WD score is negative, which reflects person-focused team members. Such coupling relative OC and WD dimension scores get more meaning when the interaction is observed through the characteristics of person vs task focused individuals in the team. For example, when the person-focused style is present in team explorers, their verbal engagement will be reduced as they tend to ensure that all concerned are heard. On the other hand, when the task-focused style is present, they will complement their explorative with a tendency for strong leadership, thus engage even more.

The direct effect of WD dimension has been identified on team-level, where the correlation analysis has shown a significant $(\mathrm{p}=0.0573)$ and high, positive correlation $(0.798)$ between the average WD score and the average verbal rate of team members. The results are aligned with the assumption that taskfocused teams will have higher levels of verbal engagement than person-focused teams, due to the tendency for high task commitment and developing clear action plans when generating ideas.

\section{Conclusion and further directions}

The problem-solving perspective of conceptual design employed throughout this paper provided a more comprehensive understanding of the verbal engagement in a conceptual design task. The results of the protocol analysis revealed that team members with the lowest OC score tend to have higher verbal engagement. Also, this effect is amplified if they also have a higher WD score. No significant effect was found between MP score and verbal engagement. Furthermore, it has been observed that teams with higher average WD score have higher average verbal engagement.

The findings must not be generalised due to the limited sample size (both on individual and team level), and due to the single size of teams and type of design task used. Also, the ongoing validation of the VIEW framework might result in some minor framework changes. Thus, the results rather point to specifics that require further rigorous investigation. Studies with larger sample size, different team sizes and different conceptual design tasks should be conducted to investigate the role of VIEW dimensions of problem-solving when teams solve conceptual design tasks.

Next steps will study the content of team sessions, such as the number of ideas, questions and decisions, but also turn transitions between team members and the effect of differences in problem-solving style on team performance. Another research stream will be to study the same measurable parameters on the distributed teams and compare them with the studies conducted on collocated teams.

\section{Acknowledgment}

This paper reports on work funded by the Croatian Science Foundation MInMED project (www.minmed.org).

\section{References}

Andreasen, M.M. and Hein, L. (1987), Integrated Product Development, IFS, Bedford.

Andreasen, M.M., Hansen, C.T. and Cash, P. (2015), Conceptual Design: Interpretations, Mindset and Models, Springer, Cham. https://doi.org/10.1007/978-3-319-19839-2

Badke-Schaub, P. and Buerschaper, C. (2001), "Creativity and Complex Problem Solving in the Social Context", In: Allwood, C.M. and Selart, M. (Eds.), Decision Making: Social and Creative Dimensions, Springer, Dordrecht, pp. 177-196. https://doi.org/10.1007/978-94-015-9827-9_9 
Birdi, K., Leach, D. and Magadley, W. (2016), “The Relationship of Individual Capabilities and Environmental Support with Different Facets of Designers' Innovative Behavior", Journal of Product Innovation Management, Vol. 33 No. 1, pp. 19-35. https://doi.org/10.1111/jpim.12250

Carr, P.G., de la Garza, J.M. and Vorster, M.C. (2002), "Relationship between Personality Traits and Performance for Engineering and Architectural Professionals Providing Design Services", Journal of Management in Engineering, Vol. 18 No. 4, pp. 158-166. https://doi.org/10.1061/(ASCE)0742-597X(2002)18:4(158)

Cash, P., Hicks, B. and Culley, S. (2013), "A comparison of designer activity using core design situations in the laboratory and practice", Design Studies, Vol. 34 No. 5, pp. 575-611. https://doi.org/10.1016/j.destud.2013.03.002

Cross, N. (2001), "Design cognition: results from protocol and other empirical studies of design activity", In: Eastman, C.M., McCracken, W.M. and Newstetter, W.C. (Eds.), Design Knowing and Learning: Cognition in Design Education, Elsevier Science, pp. 79-103. https://doi.org/10.1016/B978-008043868-9/50005-X

Dasgupta, N., Scircle, M.M. and Hunsinger, M. (2015), "Female peers in small work groups enhance women's motivation, verbal participation, and career aspirations in engineering", Proceedings of the National Academy of Sciences, Vol. 112 No. 16, pp. 4988-4993. https://doi.org/10.1073/pnas.1422822112

Dieter, G.E. and Schmidt, L.C. (2013), Engineering Design, 5th ed., McGraw-Hill Education, New York.

DiMicco, J.M., Pandolfo, A. and Bender, W. (2004), "Influencing group participation with a shared display", Proceedings of the 2004 ACM Conference on Computer Supported Cooperative Work (CSCW '04), Chicago, Illinois, USA, November 6-10, 2004, ACM, New York, pp. 614-623. https://doi.org/10.1145/1031607.1031713

Dinar, M., Shah, J.J., Cagan, J., Leifer, L., Linsey, J. et al. (2015), “Empirical Studies of Designer Thinking: Past, Present, and Future", Journal of Mechanical Design, Vol. 137 No. 2, pp. 021101. https://doi.org/10.1115/1.4029025

Dorst, K. (2011), “The core of 'design thinking' and its application”, Design Studies, Vol. 32 No. 6, pp. 521-532. https://doi.org/10.1016/j.destud.2011.07.006

Eisentraut, R. (1999), "Styles of problem solving and their influence on the design process", Design Studies, Vol. 20 No. 5, pp. 431-437. https://doi.org/10.1016/S0142-694X(99)00016-2

Goldschmidt, G. (2014), Linkography: Unfolding the Design Process, The MIT Press, Cambridge. https://doi.org/10.1162/DESI_r_00329

Hansen, C.T., Dorst, K. and Andreasen, M.M. (2009), "Problem formulation as a discursive design activity", Proceedings of the 17th International Conference on Engineering Design (ICED '09), Vol. 1: Design Processes, Stanford, California, USA, August 24-27, 2009, The Design Society, Glasgow, pp. 145-156.

Isaksen, S.G. and Geuens, D. (2006), A Technical Report of the Relationships Between an Assessment of Problem Solving Style and Creative Problem Solving, Creative Problem Solving Group, Inc., Orchard Park, New York.

Jablokow, K.W. (2005), "The catalytic nature of science: Implications for scientific problem solving in the $21 \mathrm{st}$ century", Technology in Society, Vol. 27 No. 4, pp. 531-549. https://doi.org/10.1016/j.techsoc.2005.08.006

Jablokow, K.W., Teerlink, W., Yilmaz, S., Daly, S.R. and Silk, E.M. (2015), “The impact of teaming and cognitive style on student perceptions of design ideation outcomes", 122nd ASEE Annual Conference \& Exposition, Conference Proceedings, Making Value for Society, Seattle, Washington, USA, June 14-17, 2015, pp. 11855. https://doi.org/10.18260/p.24885

Kirton, M.J. (2003), Adaption-Innovation: In the Context of Diversity and Change, Routledge, London. https://doi.org/10.4324/9780203695005

Kuipers, B.S., Higgs, M.J., Tolkacheva, N.V. and de Witte, M.C. (2009), "The Influence of Myers-Briggs Type Indicator Profiles on Team Development Processes", Small Group Research, Vol. 40 No. 4, pp. 436-464. https://doi.org/10.1177/1046496409333938

Kurakawa, K. (2004), “A scenario-driven conceptual design information model and its formation”, Research in Engineering Design, Vol. 15 No. 2, pp. 122-137. https://doi.org/10.1007/s00163-004-0050-z

Lawson, B. and Dorst, K. (2009), Design Expertise, 1st ed., Routledge, London. https://doi.org/10.4324/9781315072043

Liikkanen, L.A. and Perttula, M. (2009), "Exploring problem decomposition in conceptual design among novice designers", Design Studies, Vol. 30 No. 1, pp. 38-59. https://doi.org/10.1016/j.destud.2008.07.003

Macmillan, S., Steele, J., Austin, S., Kirby, P. and Spencea, R. (2001), "Development and verification of a generic framework for conceptual design”, Design Studies, Vol. 22 No. 2, pp. 169-191. https://doi.org/10.1016/S0142694X(00)00025-9

Nik Ahmad Ariff, N.S., Badke-Schaub, P., Eris, O. and Suib, S.S.S. (2012), “A framework for reaching common understanding during sketching in design teams", Proceedings of the DESIGN 2012 / 12th International Design Conference, Dubrovnik, Croatia, May 21-24, 2012, The Design Society, Glasgow, pp. 1525-1533.

Ohland, M.W., Sheppard, S.D., Lichtenstein, G., Eris, O., Chachra, D. and Layton, R. (2008), "Persistence, Engagement, and Migration in Engineering Programs", Journal of Engineering Education, Vol. 97 No. 3, pp. 259-278. https://doi.org/10.1002/j.2168-9830.2008.tb00978.x 
Pahl, G. and Beitz, W. (1996), Engineering Design: A Systematic Approach, Springer, London. https://doi.org/10.1007/978-1-4471-3581-4

Peeters, M.A.G., Rutte, C.G., van Tuijl, H.F.J.M. and Reymen, I.M.M.J. (2008), "Designing in teams: Does personality matter?", Small Group Research, Vol. 39 No. 4, pp. 438-467. https://doi.org/10.1177/1046496408319810

Philipsen, G.F. and Saine, T.J. (1973), "The effect of reward criteria on verbal participation in group discussion", Speech Monographs, Vol. 40 No. 2, pp. 151-153. https://doi.org/10.1080/03637757309375790

Schön, D. (1983), The Reflective Practitioner: How Professional Think in Action, 1st ed., Basic Books, New York.

Selby, E.C., Treffinger, D.J. and Isaksen, S.G. (2014), Foundations of VIEW, Creative Problem Solving Group, Inc., Orchard Park, New York.

Sonalkar, N., Jablokow, K., Edelman, J., Mabogunje, A. and Leifer, L. (2017), "Design Whodunit: The Relationship Between Individual Characteristics and Interaction Behaviors in Design Concept Generation", ASME 2017 International Design Engineering Technical Conferences and Computers and Information in Engineering Conference, Volume 7: 29th International Conference on Design Theory and Methodology, Cleveland, Ohio, USA, August 6-9, 2017, American Society of Mechanical Engineers (ASME), New York, pp. V007T06A009. https://doi.org/10.1115/DETC2017-68239

Sonalkar, N., Mabogunje, A. and Leifer, L. (2013), "Developing a visual representation to characterize momentto-moment concept generation in design teams", International Journal of Design Creativity and Innovation, Vol. 1 No. 2, pp. 93-108. https://doi.org/10.1080/21650349.2013.773117

Sorrentino, R.M. and Field, N. (1986), "Emergent leadership over time: The functional value of positive motivation.", Journal of Personality and Social Psychology, Vol. 50 No. 6, pp. 1091-1099. https://doi.org/10.1037/0022-3514.50.6.1091

Stempfle, J. and Badke-Schaub, P. (2002), "Thinking in design teams - an analysis of team communication", Design Studies, Vol. 23 No. 5, pp. 473-496. https://doi.org/10.1016/S0142-694X(02)00004-2

Takai, S. and Esterman, M. (2017), "Towards a Better Design Team Formation: A Review of Team Effectiveness Models and Possible Measurements of Design-Team Inputs, Processes, and Outputs", ASME 2017 International Design Engineering Technical Conferences and Computers and Information in Engineering Conference, Volume 3: 19th International Conference on Advanced Vehicle Technologies; 14th International Conference on Design Education; 10th Frontiers in Biomedical Devices, Cleveland, Ohio, USA, August 6-9, 2017, American Society of Mechanical Engineers (ASME), New York, pp. V003T04A018. https://doi.org/10.1115/DETC2017-68091

Treffinger, D.J., Isaksen, S.G. and Selby, E.C. (2014), Evidence Supporting VIEW, Creative Problem Solving Group, Inc., Orchard Park, New York.

Treffinger, D.J., Selby, E.C. and Isaksen, S.G. (2008), "Understanding individual problem-solving style: A key to learning and applying creative problem solving”, Learning and Individual Differences, Vol. 18 No. 4, pp. 390401. https://doi.org/10.1016/j.lindif.2007.11.007

Ullman, D.G. (2010), The Mechanical Design Process, 4th ed., McGraw-Hill Higher Education, Boston.

Varvel, T., Adams, S.G., Pridie, S.J. and Ruiz Ulloa, B.C. (2004), "Team Effectiveness and Individual MyersBriggs Personality Dimensions”, Journal of Management in Engineering, Vol. 20 No. 4, pp. 141-146. https://doi.org/10.1061/(ASCE)0742-597X(2004)20:4(141)

Willard, D. and Strodtbeck, F.L. (1972), "Latency of Verbal Response and Participation in Small Groups", Sociometry, Vol. 35 No. 1, pp. 161-175. https://doi.org/10.2307/2786556

Wulvik, A., Jensen, M.B. and Steinert, M. (2017), “Temporal Static Visualisation of Transcripts for Pre-Analysis of Video Material: Identifying Modes of Information Sharing”, In: Christensen, B.T., Ball, L.J. and Halskov, K. (Eds.), Analysing Design Thinking: Studies of Cross-Cultural Co-Creation, CRC Press/Balkema, Leiden, pp. 271-287. https://doi.org/10.1201/9781315208169-19

Yan, Y., Childs, P.R.N. and Hall, A. (2013), “An assessment of personality traits and their implication for creativity amongst Innovation Design Engineering masters students using the MBTI and KTS instruments", Proceedings of the 19th International Conference on Engineering Design (ICED '13), Vol. 7: Human Behaviour in Design, Seoul, Korea, August 19-22, 2013, The Design Society, Glasgow, pp. 317-326.

Tomislav Martinec, mag. ing. mech.

University of Zagreb, Faculty of Mechanical Engineering and Naval Architecture

Ivana Lučića 5, 10000 Zagreb, Croatia

Email: tomislav.martinec@fsb.hr 